\title{
Tandem Phosphorothioate Modifications for DNA Adsorption Strength and Polarity Control on Gold Nanoparticles
}

\author{
Wenhu Zhou ${ }^{1,2}$, Feng Wang ${ }^{2}$, Jinsong Ding ${ }^{1 *}$ and Juewen Liu ${ }^{1,2 *}$ \\ 1. School of Pharmaceutical Sciences, Central South University, Changsha, Hunan, China, \\ 410013. \\ Email: dingjs0221@163.com \\ 2. Department of Chemistry, Waterloo Institute for Nanotechnology, University of Waterloo, \\ Waterloo, Ontario, Canada, N2L 3G1. \\ *Email: liujw@uwaterloo.ca
}

This document is the Accepted Manuscript version of a Published Work that appeared in final form in Applied Materials \& Interfaces, copyright $@$ American Chemical Society after peer review and technical editing by publisher. To access the final edited and published work see Zhou, W., Wang, F., Ding, J., \& Liu, J. (2014). Tandem Phosphorothioate Modifications for DNA Adsorption Strength and Polarity Control on Gold Nanoparticles. ACS Applied Materials \& Interfaces, 6(17), 14795-14800. https://doi.org/10.1021/ am504791b 


\begin{abstract}
Unmodified DNA was recently used to functionalize gold nanoparticles via DNA base adsorption. Compared to thiolated DNA, however, the application of unmodified DNA is limited by the lack of sequence generality, adsorption polarity control and poor adsorption stability. We report that these problems can be solved using phosphorothioate (PS) DNA. PS DNA binds to gold mainly via the sulfur atom and is thus less sequence dependent. The adsorption affinity is ranked to be thiol > PS > adenine > thymine. Tandem PS improves adsorption strength, allows tunable DNA density, and the resulting conjugates are functional at a low cost.
\end{abstract}

Keywords: DNA, gold nanoparticles, phosphorothioate, adsorption, self-assembly, nanotechnology 
As a structure-directing and functional polymer, DNA has found numerous applications in analytical, materials and biomedical sciences in the past few decades. ${ }^{1-3}$ For example, many novel nanostructures were created based on DNA programmable assembly. ${ }^{4-6}$ Interfacing DNA with inorganic nanomaterials is a key step of bottom-up fabrication to produce functional hybrids and devices. ${ }^{7-9}$ In particular, DNA-directed assembly of gold nanoparticles (AuNPs) has tremendously fueled the growth of nanobiotechnology. ${ }^{10-16}$ The dense layer of DNA creates a unique colloidal system with many fundamentally interesting physical properties. ${ }^{10}$

End-labeled thiol is the main anchor for linking DNA to AuNPs due to strong Au-thiol interactions. Various methods were developed to optimize this conjugate chemistry. ${ }^{17}$ Recently, non-thiolated DNA has also been explored to functionalize AuNPs with the advantages of having a better control over DNA density, conformation and hybridization kinetics. ${ }^{18-21}$ These features are crucial for practical applications but they are comparatively more difficult to achieve with thiolated DNA.

Attaching non-thiolated DNA relies on DNA base adsorption. The four nucleobases have different adsorption affinity towards gold. Using a displacement assay, Tarlov, Whitman and coworkers observed the following adsorption strength ranking for DNA homopolymers, $\mathrm{A}>\mathrm{C}>$ $\mathrm{G}>\mathrm{T}^{22}$ This is also consistent with other single base adsorption studies. ${ }^{22-24}$ Therefore, with a di-block DNA containing poly-A and poly-T blocks, the poly-A block is likely to attach to gold surface while the poly-T block should be available for hybridization. By adjusting the length of the poly-A block, the DNA density is readily tunable. However, this system has two inter-related problems that hinder its broader applications. The first limitation is the lack of sequence generality. ${ }^{21}$ For example, if a DNA is rich in adenine or cytosine in the whole sequence, it is unlikely that it can be attached only via the intended poly-A anchor. ${ }^{21}$ If the other end is also 
adsorbed on gold, subsequent hybridization may be hindered. The second problem is stability. The adsorption affinity of adenine is weaker than that for thiol, and DNA might dissociate more easily under harsh conditions or after long-term storage.

Ideally, a more pronounced chemical distinction is needed to differentiate the block intended for attachment to gold from the block for hybridization. Non-thiolated DNA relies on the coordination of the adenine base (e.g. the N7 position in yellow circles, Figure 1A). We herein propose a simple modification of replacing one of the non-bridging phosphate oxygen atoms by sulfur (i.e. phosphorothioate or PS modification, Figure 1B). Since both the sulfur and adenine could contribute to binding, the overall affinity might be stronger. If such PS modifications are made in tandem, much higher stability is expected due to polyvalent interactions. Since PS modifications are made within the DNA backbone, the advantages of nonthiolated DNA should still be maintained. PS DNA has been used for various applications. The best known example is probably for anti-sense DNA to be more resistant to nucleases. ${ }^{25}$ It is also used for assembling metallic nanoparticles, ${ }^{26-28}$ proteins, ${ }^{29}$ or semiconductor quantum dots, ${ }^{30,31}$ and for biosensor development. ${ }^{32,33}$ PS modification is valuable for studying enzyme mechanism as well. ${ }^{34-36}$ It needs to be noted that the cost of a PS modification is only $~ 3 \%$ of that of a thiol, which makes it more affordable to researchers. 

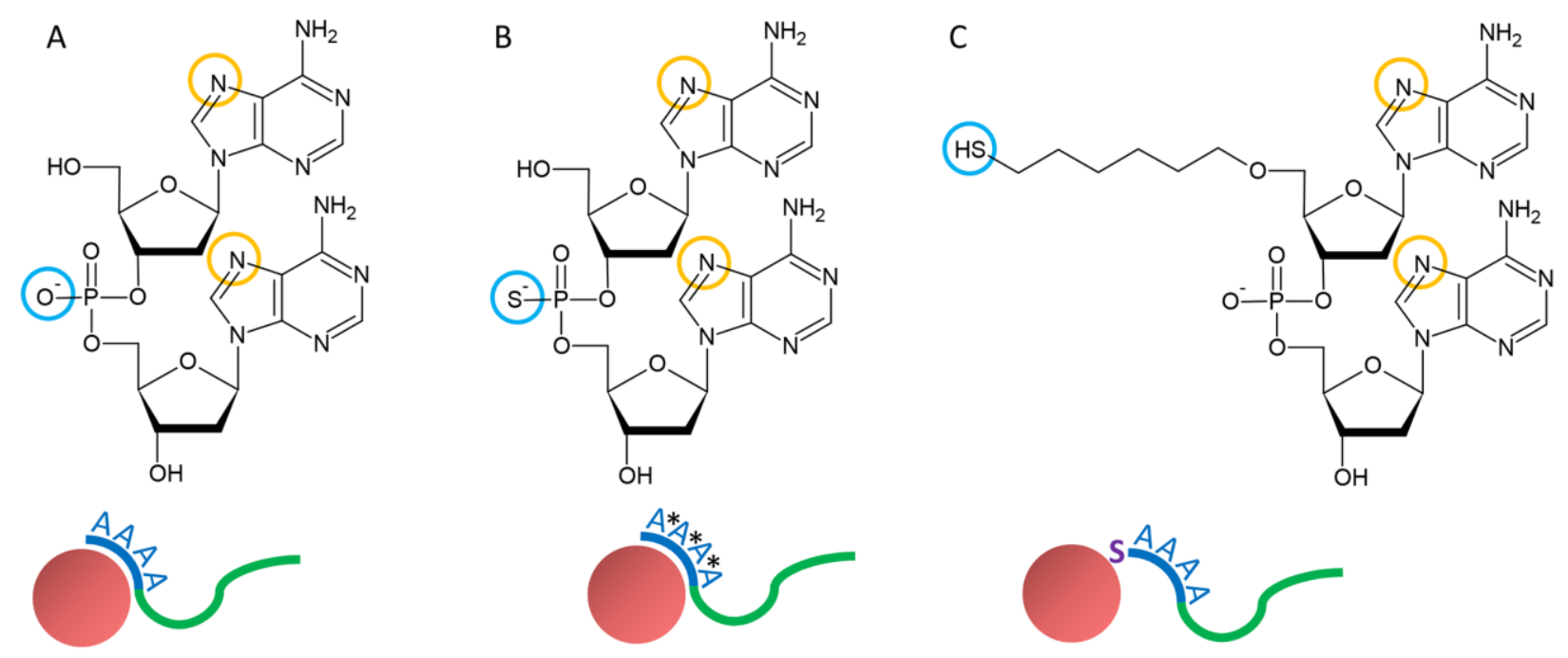

Figure 1. Schematics of three methods of attaching DNA to AuNPs. (A) Unmodified DNA with a phosphate backbone via base adsorption. (B) PS DNA. (C) Thiolated (SH) DNA. The N7 postion of the adenines are circled in yellow, which is a high affinity site for the adenine coordination to gold. The phosphate oxygen, and the PS and thiol modifications are circled in blue. For clarity of the figure, only one DNA strand is shown on each AuNP. The green block is intended for hybridization.

Table 1. DNA sequences used in this work. The PS modification is denoted by *.

\begin{tabular}{|c|c|}
\hline Name & Sequences and modifications (from $5^{\prime}$ to $3^{\prime}$ ) \\
\hline DNA1 & A*ACCCAGGTTCTCT \\
\hline DNA2 & $\mathrm{A} * \mathrm{~A} * \mathrm{~A} * \mathrm{~A} * \mathrm{ACCCAGGTTCTCT}$ \\
\hline DNA3 & $\mathrm{A} * \mathrm{~A} * \mathrm{~A} * \mathrm{~A} * \mathrm{~A} * \mathrm{~A} * \mathrm{~A} * \mathrm{~A} * \mathrm{ACCCAGGTTCTCT}$ \\
\hline DNA4 & A*A*A*A*A*A*A*A*A*A*A*A*ACCCAGGTTCTCT \\
\hline DNA5 & A*A*A*A*A*A*A*A*A*A*A*A*A*A*A*A*ACCCAGGTTCTCT \\
\hline
\end{tabular}




\begin{tabular}{l|l}
\hline DNA6 & AAAAAAAAACCCAGGTTCTCT-FAM \\
\hline DNA7 & A*A*A*A*A*A*A*A*ACCCAGGTTCTCT-FAM \\
\hline DNA8 & SH-AAAAAAAAACCCAGGTTCTCT-FAM \\
\hline DNA9 & SH-AAAAAAAAACCCAGGTTCTCT \\
\hline DNA10 & AAAAAAAAACCCAGGTTCTCT \\
\hline DNA11 & A*AAAAAAAACCCAGGTTCTCT \\
\hline DNA12 & A*A*AAAAAAACCCAGGTTCTCT \\
\hline DNA13 & A*A*A*A*AAAAACCCAGGTTCTCT \\
\hline DNA14 & A*A*A*A*A*A*AAACCCAGGTTCTCT \\
\hline DNA15 & T*T*T*T*T*T*T*T*TCCCAGGTTCTCT \\
\hline DNA16 & TCACAGATGCGTAAAAAAAAA \\
\hline DNA17 & TCACAGATGCGTA*A*A*A*A*A*A*A*A \\
\hline DNA18 & ACGCATCTGTGAAGAGAACCTGGG \\
\hline
\end{tabular}

In Figure 1, the three strategies for attaching DNA to AuNPs are presented. For systematic comparison, we designed a 21-mer DNA with a block of 9-adenine on the 5'-end to attach to AuNPs. The rest 12 nucleotides are a random sequence for hybridization (DNA6, see Table 1 for DNA sequence). DNA6 is a normal DNA without any modifications (also called PO DNA). The PS DNA (DNA7) was modified at each phosphate in the poly-A block and it contained 8 PS. A thiolated (SH) DNA was also used for comparison (DNA8). All the three DNAs contained a FAM (6-carboxyfluorescein) label at the 3'-end. A low pH DNA loading method was used to attach DNA to $13 \mathrm{~nm}$ AuNPs followed by adjusting $\mathrm{pH}$ to neutral and 
further incubation. ${ }^{37,38}$ We measured the density of DNA on AuNPs in all the three samples (Figure 2A). The thiolated DNA has the highest density of $\sim 80$ DNA per AuNP, followed by the PS DNA ( 60$)$ and the PO DNA ( 50$)$. AuNPs functionalized with the PS and the PO DNA migrated as a sharp band in gel electrophoresis, confirming stable DNA attachment (Figure S1). The UV-vis spectra of the samples were measured and they all showed a sharp surface plasmon peak at $520 \mathrm{~nm}$, indicating that the conjugates retained colloidal stability in the preparation process (Figure 2B). The dynamic light scattering (DLS) spectrum of the citrate-capped AuNPs gives a size of $\sim 13 \mathrm{~nm}$ (Figure $2 \mathrm{C}$ ), which increased to $\sim 16 \mathrm{~nm}$ after adding the DNAs. This size increase is attributed to the attached DNA layer and it further confirms the stability of these conjugates.
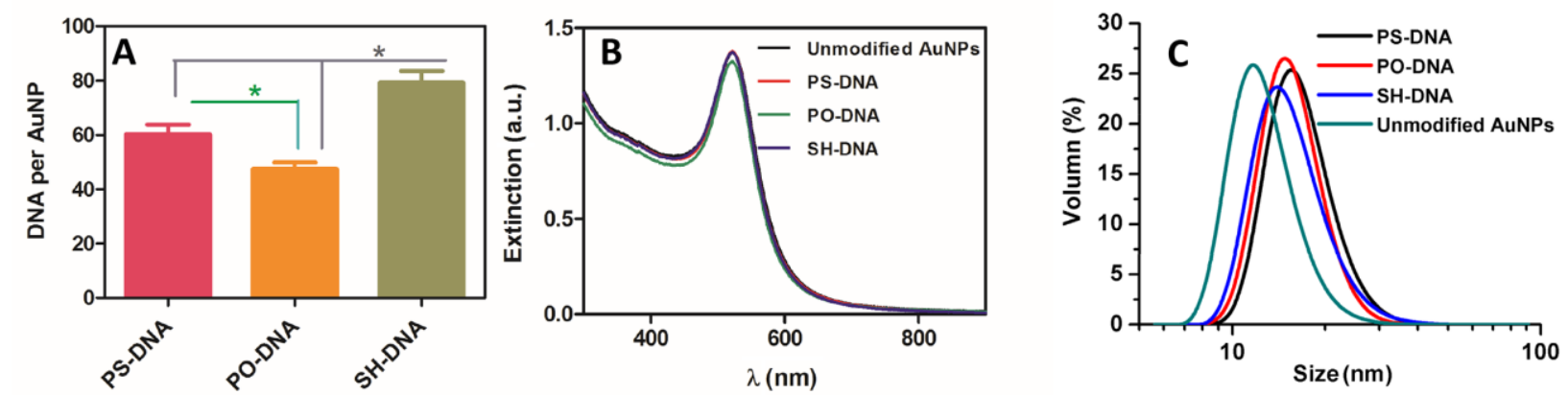

Figure 2. (A) DNA loading capacity of PS, PO and SH-DNA. Characterization of the DNA functionalized AuNPs using (B) UV-vis spectroscopy and (C) DLS.

Since the main assumption of this work is that PS-modified DNA attach to AuNPs more strongly than the PO counterpart, this was tested first. The conjugate stability was assayed in two aspects. First, to study DNA adsorption stability, ${ }^{39,40}$ we used glutathione (GSH) as a probe, which is a thiol containing tripeptide found in cells. Since AuNPs are fluorescence quenchers, 
addition of GSH might displace the adsorbed FAM-labeled DNAs and thus increase fluorescence. No fluorescence increase was observed for all the three samples after 25 min incubation in buffer, suggesting that they are stable in normal conditions (Figure 3A). Upon addition of GSH at 25 min, the PO DNA released the fastest while the PS and SH DNA showed similarly slower release. We further used mercaptohexanol as a probe and the SH DNA adsorbed more strongly than the PS DNA (Figure S2). This confirms that PS DNA binds more strongly than PO DNA and but not as the SH-DNA.

Aside from DNA adsorption stability, another important aspect is the colloidal stability of AuNPs. For this, salt was used as a probe. We first incubated the AuNPs in $700 \mathrm{mM} \mathrm{NaCl}$ and the PO DNA (DNA10) capped AuNPs turned to purple color, indicating the loss of colloidal stability (inset of Figure 3B). On the other hand, the PS sample (DNA3) remained stable. This stability is sufficient for most analytical and biomedical applications. For quantitative comparison, we challenged the AuNPs with various concentrations of $\mathrm{Mg}^{2+}$ and monitored the color by UV-vis spectroscopy (Figure 3B). An increase in the ratio of extinction at $650 \mathrm{~nm}$ over $520 \mathrm{~nm}$ indicates color change to blue and aggregation of AuNPs. ${ }^{41}$ Divalent $\mathrm{Mg}^{2+}$ ions are much more efficient in screening charge repulsion and can cause AuNP aggregation at lower concentrations. The PO DNA capped AuNPs aggregated with greater than $2 \mathrm{mM} \mathrm{Mg}^{2+}$, while the PS DNA sample aggregated with $5 \mathrm{mM} \mathrm{Mg}^{2+}$. The SH DNA sample has the highest stability, showing no sign of aggregation. Overall, the PS DNA capped AuNPs have significantly improved stability compared to the PO DNA. To test for long-term stability, we removed free DNA and the purified conjugates were stored in buffer for 3 days. Significant aggregation was observed with $5 \mathrm{mM} \mathrm{Mg}^{2+}$ was added, suggesting that the conjugates still need to be freshly prepared (Figure S3) ${ }^{39}$ 

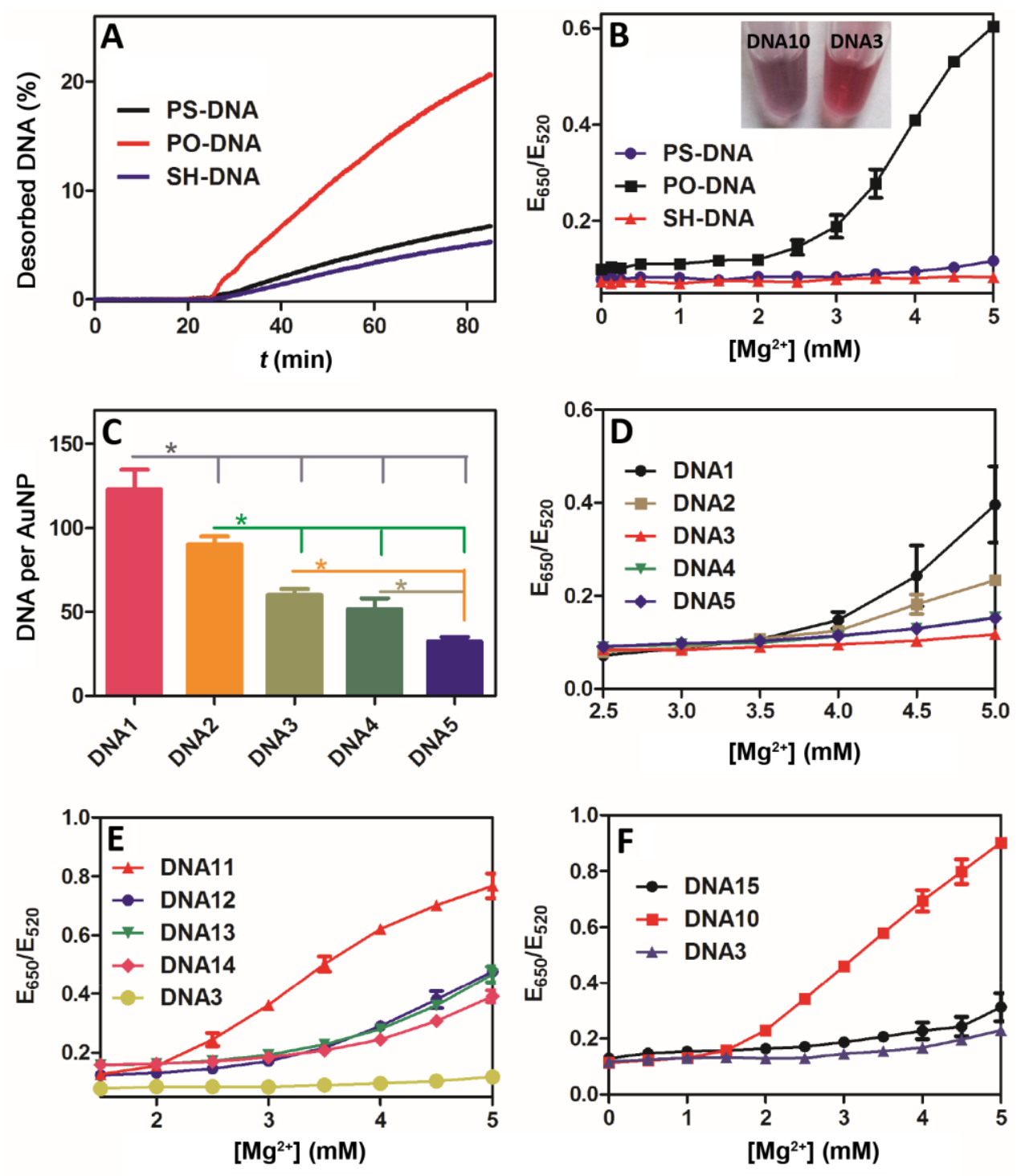

Figure 3. (A) DNA desorption kinetics for PS, PO and SH DNA (DNA 6, 7, 8) from AuNPs in the presence of $2 \mathrm{mM}$ GSH. (B) Extinction ratio $\mathrm{E}_{650} / \mathrm{E}_{520}$ as a function of $\mathrm{Mg}^{2+}$ concentration for PS, PO and SH DNA (DNA3, 9, 10). Inset: photographs of AuNPs with PO and PS DNA in the presence of $700 \mathrm{mM} \mathrm{NaCl}$. Effect of PS modified poly-A length on (C) DNA loading capacity and (D) stability of AuNPs. (E) Effect of the number of PS modifications on AuNP stability while fixing the poly-A block to be A9. (F) Stability of AuNPs as a function of DNA sequence. 
In the above studies, we used a 9-adenine ( $\left.\mathrm{A}_{9}\right)$ block for attachment to the gold. Since this length is likely to be crucial, we next systematically varied it from 2 to 17 and the number of PS modifications was from 1 to 16 (DNA1-5). The number of DNA attached to each AuNP is quantified in Figure 3C, where a longer poly-A block produced a lower DNA density. This is consistent with more adenine binding. ${ }^{19}$ Note that it is different from the attachment of DNA or polymer with a single terminal thiol. ${ }^{42}$ We next compared their colloidal stability by adding $\mathrm{Mg}^{2+}$ (Figure 3D). DNA with $\mathrm{A}_{2}$ (e.g. 2 adenines) has the lowest stability, and significant improvement was observed with $\mathrm{A}_{5}$ and $\mathrm{A}_{9}$. Further increase of the poly-A block length resulted in decreased stability. This was attributed to the decreased DNA density, reducing the steric stabilization effect. Therefore, $A_{9}$ is an optimal length to achieve both good DNA density and stability.

Next, we fixed the number of adenine to be 9 and varied the number of PS modifications (DNA3, DNA11-14). As shown in Figure 3E, the highest stability was achieved with the full 8 PS modifications. Therefore, each PS modification contributes towards stability. Finally, to test whether the adenine base contributes to binding, we studied a $T_{9}$ PS DNA for anchoring (DNA15), and it showed a similar stability profile as the A9 PS DNA (Figure 3F). Since thymine itself has very low affinity on gold, we conclude that PS is the main binder instead of the bases. This makes PS DNA adsorption less sequence dependent. This study shows that the affinity of PS to gold is weaker compared to SH but stronger than adenine binding. In addition, the polyvalent effect for tandem PS allows for much stronger stability.

After studying stability, we next tested function. As mentioned previously, one issue impeding the general application of non-thiolated DNA is the special sequence requirement. For example, for the normal PO DNA, the block intended for hybridization should be rich in T and G. Otherwise, both ends might attach to gold, preventing hybridization and reducing colloidal 
stability (Figure 4A). Since the PS DNA binding is stronger than adenine, which is the strongest base for binding to gold, the issue of polarity control of DNA attachment can be solved. The particular sequence we used was DNA16, which has four A/C bases towards the other end. As shown in Figure 4C, it has poor colloidal stability and aggregated in the presence of $400 \mathrm{mM}$ $\mathrm{NaCl}$. On the other hand, the PS modified DNA (DNA17) remained stable. This experiment confirms that the PS DNA strategy has expanded the range of DNA sequences that can be used for attachment.

Next, we prepared two types of PS DNA functionalized AuNPs (with DNA3 and DNA17, respectively) for DNA-directed assembly (Figure 4B). The PS-modified AuNPs showed fast color change upon adding the linker DNA, as indicated by the decrease of the extinction ratio (Figure 4E). At the same time, the particles remained stable in the absence of the linker. The aggregates showed reversible behavior (Figure 4D), where the color of the sample returned to red upon heating to $75{ }^{\circ} \mathrm{C}$ and became purple again upon cooling. We measured the melting transition of PS DNA sample and we observed a characteristically sharp melting transition (Figure 4F). 

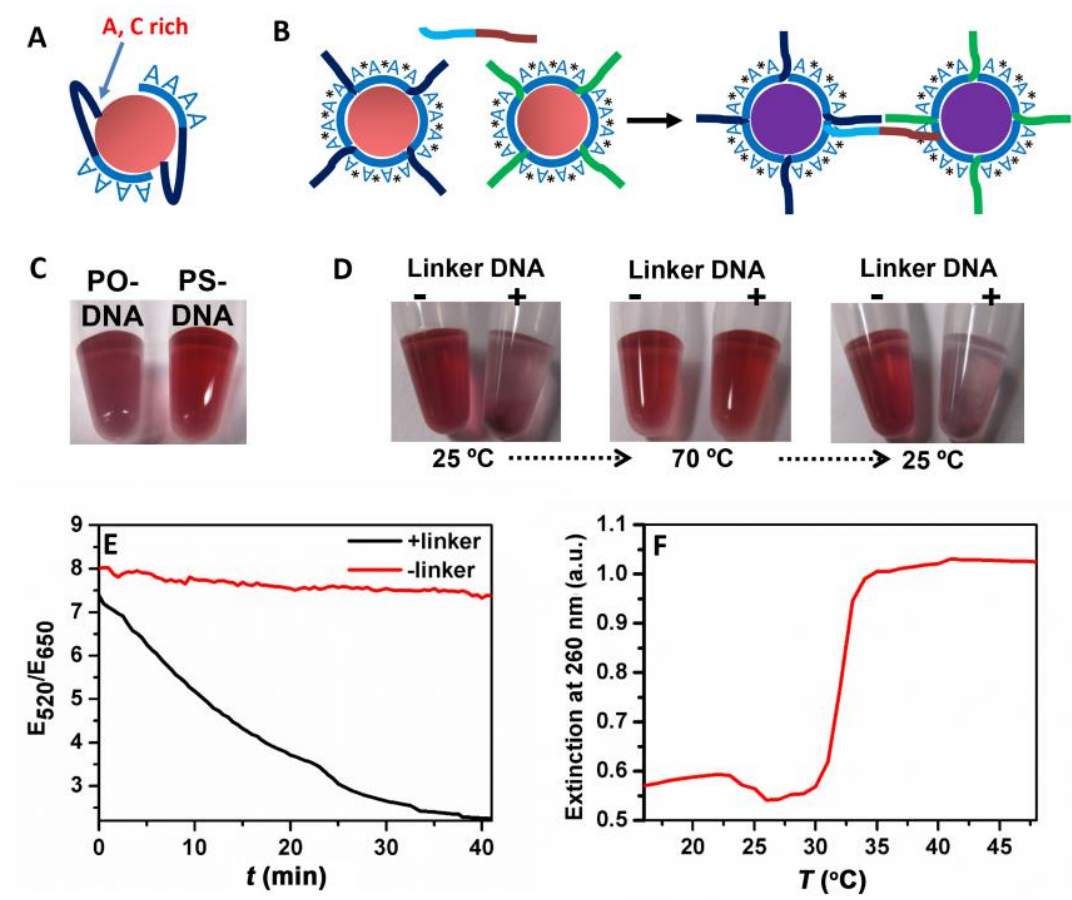

Figure 4. (A) Scheme of PO DNA attachment to AuNPs via both ends, hindering DNA hybridization. (B) Scheme of DNA-directed assembly with PS modified DNA, where the PS containing block always attaches to the gold surface. (C) Colloidal stability of AuNPs functionalized with DNA16 (PO) and DNA17 (PS) in $400 \mathrm{mM} \mathrm{NaCl}$. (D) Reversible aggregation and melting of DNA-linked AuNPs with PS DNA. (E) Kinetics of PS DNA-functionalized AuNP color change with or without linker DNA. (F) A melting curve of DNA-linked PS DNA functionalized AuNPs.

In summary, we demonstrated that tandem PS DNA forms highly stable conjugates with AuNPs. This allows selective attachment of DNA through rational sequence design and it can be applied to many more sequences than the normal PO DNA. PS modification allows much more stable DNA adsorption compared to the unmodified PO DNA and also confers better AuNP 
colloidal stability. At the same time, PS DNA also maintains the advantages of PO DNA. For example, it allows the tuning of DNA density by changing the length of the poly-A block, which is more difficult to achieve with the thiolated DNA. In addition, PS modification is much more cost-effective than thiol. On the fundamental side, for the first time, we systematically compared the adsorption strength of thiol, DNA bases and PS. Considering the simplicity and minimal perturbation of DNA structure, PS DNA will find many applications in nanotechnology, materials science and analytical chemistry.

\section{Acknowledgement}

This work is supported by the University of Waterloo, the Natural Sciences and Engineering Research Council of Canada, Foundation for Shenghua Scholar of Central South University and the National Natural Science Foundation of China (Grant No. 21301195).

Supporting Information. Materials and methods, gel electrophoresis and additional stability data. This material is available free of charge via the Internet at http://pubs.acs.org."

\section{References}

(1) Seeman, N. C., DNA in a Material World. Nature 2003, 421, 427-431.

(2) Rosi, N. L.; Mirkin, C. A., Nanostructures in Biodiagnostics. Chem. Rev. 2005, 105, $1547-1562$.

(3) Pinheiro, A. V.; Han, D.; Shih, W. M.; Yan, H., Challenges and Opportunities for Structural DNA Nanotechnology. Nat. Nanotechnol. 2011, 6, 763-772. 
(4) Park, S. Y.; Lytton-Jean, A. K. R.; Lee, B.; Weigand, S.; Schatz, G. C.; Mirkin, C. A., DNA-Programmable Nanoparticle Crystallization. Nature 2008, 451, 553-556.

(5) Nykypanchuk, D.; Maye, M. M.; van der Lelie, D.; Gang, O., DNA-Guided Crystallization of Colloidal Nanoparticles. Nature 2008, 451, 549-552.

(6) Aldaye, F. A.; Palmer, A. L.; Sleiman, H. F., Assembling Materials with DNA as the Guide. Science 2008, 321, 1795-1799.

(7) Zhang, T.; Yang, Z.; Liu, D., DNA Discrete Modified Gold Nanoparticles. Nanoscale 2011, 3, 4015-4021.

(8) Cheng, W. L.; Campolongo, M. J.; Cha, J. J.; Tan, S. J.; Umbach, C. C.; Muller, D. A.; Luo, D., Free-Standing Nanoparticle Superlattice Sheets Controlled by DNA. Nat. Mater. 2009, 8, 519-525.

(9) Katz, E.; Willner, I., Nanobiotechnology: Integrated Nanoparticle-Biomolecule Hybrid Systems: Synthesis, Properties, and Applications. Angew. Chem., Int. Ed. 2004, 43, 60426108.

(10) Cutler, J. I.; Auyeung, E.; Mirkin, C. A., Spherical Nucleic Acids. J. Am. Chem. Soc. 2012, 134, 1376-1391.

(11) Liu, J.; Cao, Z.; Lu, Y., Functional Nucleic Acid Sensors. Chem. Rev. 2009, 109, 19481998.

(12) Li, D.; Song, S. P.; Fan, C. H., Target-Responsive Structural Switching for Nucleic AcidBased Sensors. Acc. Chem. Res. 2010, 43, 631-641.

(13) Zhao, W.; Brook, M. A.; Li, Y., Design of Gold Nanoparticle-Based Colorimetric Biosensing Assays. ChemBioChem 2008, 9, 2363-2371. 
(14) Wang, H.; Yang, R. H.; Yang, L.; Tan, W. H., Nucleic Acid Conjugated Nanomaterials for Enhanced Molecular Recognition. ACS Nano 2009, 3, 2451-2460.

(15) Wei, M.; Chen, N.; Li, J.; Yin, M.; Liang, L.; He, Y.; Song, H. Y.; Fan, C. H.; Huang, Q., Polyvalent Immunostimulatory Nanoagents with Self-Assembled CpG OligonucleotideConjugated Gold Nanoparticles. Angew. Chem. Int. Ed. 2012, 51, 1202-1206.

(16) Zhao, Z.; Jacovetty, E. L.; Liu, Y.; Yan, H., Encapsulation of Gold Nanoparticles in a DNA Origami Cage. Angew. Chem. Int. Ed. 2011, 50, 2041-2044.

(17) Mirkin, C. A.; Letsinger, R. L.; Mucic, R. C.; Storhoff, J. J., A DNA-Based Method for Rationally Assembling Nanoparticles into Macroscopic Materials. Nature 1996, 382, 607-609.

(18) Opdahl, A.; Petrovykh, D. Y.; Kimura-Suda, H.; Tarlov, M. J.; Whitman, L. J., Independent Control of Grafting Density and Conformation of Single-Stranded DNA Brushes. Proc. Natl. Acad. Sci. U.S.A. 2007, 104, 9-14.

(19) Pei, H.; Li, F.; Wan, Y.; Wei, M.; Liu, H.; Su, Y.; Chen, N.; Huang, Q.; Fan, C., Designed Diblock Oligonucleotide for the Synthesis of Spatially Isolated and Highly Hybridizable Functionalization of DNA-Gold Nanoparticle Nanoconjugates. J. Am. Chem. Soc. 2012, 134, 11876-11879.

(20) Zhang, X.; Liu, B.; Dave, N.; Servos, M. R.; Liu, J., Instantaneous Attachment of an Ultrahigh Density of Nonthiolated DNA to Gold Nanoparticles and Its Applications. Langmuir 2012, 28, 17053-17060.

(21) Zhang, X.; Liu, B.; Servos, M. R.; Liu, J., Polarity Control for Nonthiolated DNA Adsorption onto Gold Nanoparticles. Langmuir 2013. 
(22) Kimura-Suda, H.; Petrovykh, D. Y.; Tarlov, M. J.; Whitman, L. J., Base-Dependent Competitive Adsorption of Single-Stranded DNA on Gold. J. Am. Chem. Soc. 2003, 125, 9014-9015.

(23) Storhoff, J. J.; Elghanian, R.; Mirkin, C. A.; Letsinger, R. L., Sequence-Dependent Stability of DNA-Modified Gold Nanoparticles. Langmuir 2002, 18, 6666-6670.

(24) Liu, J., Adsorption of DNA onto Gold Nanoparticles and Graphene Oxide: Surface Science and Applications. Phys. Chem. Chem. Phys. 2012, 14, 10485-10496.

(25) Deleavey, Glen F.; Damha, M. J., Designing Chemically Modified Oligonucleotides for Targeted Gene Silencing. Chem. Biol. 2012, 19, 937-954.

(26) Lee, J. H.; Wernette, D. P.; Yigit, M. V.; Liu, J.; Wang, Z.; Lu, Y., Site-Specific Control of Distances between Gold Nanoparticles Using Phosphorothioate Anchors on DNA and a Short Bifunctional Molecular Fastener. Angew. Chem. Int. Ed. 2007, 46, 9006-9010.

(27) Pal, S.; Sharma, J.; Yan, H.; Liu, Y., Stable Silver Nanoparticle-DNA Conjugates for Directed Self-Assembly of Core-Satellite Silver-Gold Nanoclusters. Chem. Commun. 2009, 6059-6061.

(28) Kumar, A.; Phadtare, S.; Pasricha, R.; Guga, P.; Ganesh, K. N.; Sastry, M., Assembling Gold Nanoparticles in Solution Using Phosphorothioate DNA as Structural Interconnects. Curr. Sci. 2003, 84, 71-74.

(29) Lee, J. H.; Wong, N. Y.; Tan, L. H.; Wang, Z. D.; Lu, Y., Controlled Alignment of Multiple Proteins and Nanoparticles with Nanometer Resolution Via Backbone-Modified Phosphorothioate DNA and Bifunctional Linkers. J. Am. Chem. Soc. 2010, 132, 89068908. 
(30) Ma, N.; Sargent, E. H.; Kelley, S. O., One-Step DNA-Programmed Growth of Luminescent and Biofunctionalized Nanocrystals. Nat. Nanotechnol. 2009, 4, 121-125.

(31) Farlow, J.; Seo, D.; Broaders, K. E.; Taylor, M. J.; Gartner, Z. J.; Jun, Y.-w., Formation of Targeted Monovalent Quantum Dots by Steric Exclusion. Nat. Methods 2013, 10, $1203-1205$.

(32) Zhang, D.; Deng, M.; Xu, L.; Zhou, Y.; Yuwen, J.; Zhou, X., The Sensitive and Selective Optical Detection of Mercury(II) Ions by Using a Phosphorothioate DNAzyme Strategy. Chem. Eur. J 2009, 15, 8117-8120.

(33) Huang, P.-J. J.; Liu, J., Sensing Parts-Per-Trillion $\mathrm{Cd}^{2+}, \mathrm{Hg}^{2+}$, and $\mathrm{Pb}^{2+}$ Collectively and Individually Using Phosphorothioate DNAzymes. Anal. Chem. 2014, 86, 5999-6005.

(34) Wang, S.; Karbstein, K.; Peracchi, A.; Beigelman, L.; Herschlag, D., Identification of the Hammerhead Ribozyme Metal Ion Binding Site Responsible for Rescue of the Deleterious Effect of a Cleavage Site Phosphorothioate. Biochemistry 1999, 38, 1436314378.

(35) Nawrot, B.; Widera, K.; Wojcik, M.; Rebowska, B.; Nowak, G.; Stec, W. J., Mapping of the Functional Phosphate Groups in the Catalytic Core of Deoxyribozyme 10-23. FEBS J 2007, 274, 1062-1072.

(36) Cunningham, L. A.; Li, J.; Lu, Y., Spectroscopic Evidence for Inner-Sphere Coordination of Metal Ions to the Active Site of a Hammerhead Ribozyme. J. Am. Chem. Soc. 1998, $120,4518-4519$.

(37) Zhang, X.; Servos, M. R.; Liu, J., Fast pH-Assisted Functionalization of Silver Nanoparticles with Monothiolated DNA. Chem. Commun. 2012, 48, 10114-10116. 
(38) Zhang, X.; Servos, M. R.; Liu, J., Instantaneous and Quantitative Functionalization of Gold Nanoparticles with Thiolated DNA Using a pH-Assisted and Surfactant-Free Route. J. Am. Chem. Soc. 2012, 134, 7266-7269.

(39) Bhatt, N.; Huang, P.-J. J.; Dave, N.; Liu, J., Dissociation and Degradation of ThiolModified DNA on Gold Nanoparticles in Aqueous and Organic Solvents. Langmuir 2011, 27, 6132-6137.

(40) Herdt, A. R.; Drawz, S. M.; Kang, Y. J.; Taton, T. A., DNA Dissociation and Degradation at Gold Nanoparticle Surfaces. Colloids Surf., B 2006, 51, 130-139.

(41) Liu, J. W.; Lu, Y., A Colorimetric Lead Biosensor Using DNAzyme-Directed Assembly of Gold Nanoparticles. J. Am. Chem. Soc. 2003, 125, 6642-6643.

(42) Wang, W.; Wei, Q.-Q.; Wang, J.; Wang, B.-C.; Zhang, S.-H.; Yuan, Z., Role of ThiolContaining Polyethylene Glycol (Thiol-PEG) in the Modification Process of Gold Nanoparticles (Aunps): Stabilizer or Coagulant? J. Colloid Interf. Sci. 2013, 404, 223229. 